\title{
ViPer MIMO: Increasing Large MIMO Efficiency via Practical Vector-Perturbation
}

\author{
Christopher Husmann and Konstantinos Nikitopoulos \\ 5G Innovation Centre \\ Institute for Communication Systems \\ University of Surrey, Guildford, UK \\ Email: \{c.husmann, k.nikitopoulos\}@ surrey.ac.uk
}

\begin{abstract}
Large multi-user MIMO systems with spatial multiplexing are among the most promising approaches for increasing wireless throughput while serving many clients. Yet, the achievable spectral efficiency of current large MIMO systems is limited by the adoption of simple, but sub-optimal, linear precoding techniques (e.g, minimum-mean-square-error (MMSE)). Nonlinear precoding methods, like Vector Perturbation (VP), claim to be able to provide improved network throughput. However, such methods are still purely theoretical and they do not account for the practical aspects of actual wireless systems, as the corresponding complexity and latency requirements, or the need for practical rate adaptation. This paper presents ViPer, the first practical VP-based MIMO system design. ViPer substantially reduces the latency requirements of $\mathrm{VP}$ by employing massively parallel processing and realizes a practical rate adaptation method that efficiently translates VP's signal-to-noise-ratio (SNR) gains into actual throughput gains. In our first systematic experimental evaluation of VP-based precoders, we show that ViPer can deliver in practice up to $30 \%$ higher throughput than MMSE precoding with comparable latency requirements. In addition, ViPer can match the performance of state-of-the-art parallel VP precoding schemes, by utilizing less than one tenth of the processing elements.
\end{abstract}

Index Terms-Vector Perturbation, parallel processing, low latency processing

\section{INTRODUCTION}

Next generation communication systems are expected to have peak data rates $100 \times$ higher than those of current systems, to satisfy the ever-increasing demand for capacity and throughput [1]. To reach this ambitious goal not only more spectrum needs to be occupied but also the spectral efficiency has to be increased [2]. One of the most promising approaches for increasing the spectral efficiency of wireless communication systems is to use large Multiple-Input-Mutiple-Output systems with spatial multiplexing, which is expected to play a key role in upcoming cellular [3] and local-area [4] standards. This work focuses on downlink MIMO transmissions with spatial multiplexing, which is of high practical interest due to applications like high-definition video streaming [5].

Current practical designs for MIMO AP's [6], [7], employ linear precoders such as zero-forcing (ZF) [8] and minimummean-square-error [9], but their achievable rate is far from optimal for ill-conditioned channels as in the case where the number of receiving antennas approaches the number antennas at the AP side. [7], [10]. This leaves a significant amount of MIMO channel capacity unexploited [11].
In his landmark paper [12], Costa provides the capacity region of the MIMO broadcast channel. In this direction, Tomlinson-Harashima (TH) precoding [13] can significantly improve the throughput of ZF in ill-conditioned MIMO channels, but it is still far from capacity achieving. By extending TH precoding, Vector Pertubation [11] (VP) precoding further pushes the achievable data rate to be closer to the capacity. VP precoders calculate a joint perturbation vector that maximizes the receivers' signal-to-noise-ratio (SNR). Yet, to find the perturbation vector, the precoder has to solve an integer optimization problem. To do this efficiently, traditional VP precoded systems can utilize highly optimized "depth-first" sphere encoders (SE), like Geosphere [10], [14]. Still, the corresponding processing complexity and latency of sphere encoding can significantly vary, even per MIMO channel use. Moreover, its complexity and latency requirements scale exponentially with the number of concurrently transmitted information streams, making it impractical for systems supporting large numbers of users. Considering, that clock frequencies of traditional processor are plateauing [15] and that at the same time upcoming wireless systems will have higher sampling frequency and stricter latency requirements, the increased complexity of VP precoding can only be managed via efficient parallelization. The Fixed-Complexity-Sphere-Encoder (FCSE) [16] is an approximate sphere encoding algorithm that, by design, exploits parallelism and guarantees a fixed processing throughput. However, FSCE cannot adjust to the transmission channel and is unable to consistently exploit any number of available processing elements (PEs), resulting in inefficient utilization of the processing resources and in significant throughput losses as shown in Section V.

Another practical problem in VP-based precoding is how to perform efficient rate adaption. A theoretical foundation for rate adaption, in combination with VP has been laid in [17]. Yet, the rate adaption strategy has not been evaluated with practical systems and has been designed only for traditional VP precoders which, as previously discussed, are impractical for large MIMO systems.

Multisphere is a recently proposed framework for massively parallelizing the detection in large, uplink MIMO systems [18]. FlexCore [19] is an approximation of Multisphere with fixed processing throughput and latency requirements that are similar to those of linear detection methods, and has been 
shown to outperform state-of-the-art parallel decoders such as the fixed-complexity-sphere-decoder (FCSD) [20]. This is achieved by focusing the processing power to the sphere decoder tree paths that are considered to be the "most promising" to include the correct solution. However, the related "metric of promise" is based on the statistics of the noise [19] and, therefore, it is not directly applicable to VP precoding.

By extending the work of [18], [19], here we present ViPer. To the best of our knowledge, ViPer is the first practical MIMO downlink system design, that can efficiently be realized in a flexible and massively parallel manner. ViPer can exploit any number of processing elements and focuses the processing power on the most promising SE tree paths, by introducing a tailored to the downlink "metric of promise". As we show in Section V, Viper can asymptotically approach the optimal VP performance by increasing the number of available PEs. This enables the use of practical rate adaption methods adopting the framework of [17]. In Section V we show that, for the same achievable throughput, ViPer reduces the number of required PEs by a factor of up to 10 compared to state-of-art VP-based precoders. Additionally, ViPer introduces a tailored to the downlink, and applicable to all VP-based precoders, "sorted RQ decompostion" that can improve the throughput of approximate VP-based methods [16] by more than $200 \%$ or can decrease the complexity of exact VP [11] by $75 \%$. Finally we use ViPer to perform the first experimental evaluation of the "actual" gains of VP-based approaches against linear methods. We show that ViPer's throughput can exceed that of linear methods by more than $30 \%$, for similar latency requirements.

\section{SyStem MODEL}

In downlink transmission the information vector $\mathbf{u}$ that consists of the QAM-modulated information aiming all users, is first precoded vector and then $\mathbf{x}$ is transmitted. When the transmission takes place over a flat-fading MIMO channel (as in the case of an OFDM subcarrier) with $N_{t}$ transmit and $N_{r}$ receive antennas, where $N_{r} \leq N_{t}$, the received vector, that consists of the signals received from the user antennas, is given by

$$
\mathbf{y}=\mathbf{H} \cdot \mathbf{x}+\mathbf{n}
$$

with $\mathbf{H}$ being the $N_{r} \times N_{t}$ MIMO channel matrix, and with its elements $H(m, k)$ describing the transmission channel between the $m^{t h}$ receive and the $k^{t h}$ transmit antenna. The $N_{r^{-}}$ dimensional vector $\mathbf{n}$ represents the additive white Gaussian noise. To account for the finite maximum transmission power $P_{t}$ of actual APs, the power the of $N_{t}$ dimensional pre-coded vector $\mathbf{x}$ is constrained as $E\left\{\mathbf{x}^{H} \cdot \mathbf{x}\right\}=P_{t}$.

\section{Linear Percoding And Vector Pertubation}

MMSE Linear Precoder: MMSE aims to maximize the signal-to-noise-and-interference (SINR) at the user side. In general, linear pre-coding techniques generate the pre-coding vector $\mathbf{s}$ by multiplying the information vector $\mathbf{u}$ with a percoding matrix G. In the case of MMSE, the precoding matrix $\mathbf{G}_{m m s e}$ is a regularized version of channel inverse:

$$
\mathbf{G}_{m m s e}=\mathbf{H}^{H} \cdot\left(\mathbf{H} \cdot \mathbf{H}^{H}+\alpha \mathbf{I}\right)^{-1},
$$

with $\alpha$ being the normalization factor and $I$ being the identity matrix. In order not to exceed the maximum transmit power, the transmitted signal has to be be normalized by $\sqrt{\frac{P_{t}}{\gamma_{m m s e}}}$ with $\gamma_{m m s e}=E\left\{\mathbf{u}^{H} \cdot \mathbf{G}_{m m s e}^{H} \cdot \mathbf{G}_{m m s e} \cdot \mathbf{u}\right\}$. Then, the received vector $\mathbf{y}_{m m s e}$ is given by

$$
\mathbf{y}_{m m s e}=\mathbf{H} \cdot \sqrt{\frac{P_{t}}{\gamma_{m m s e}}} \cdot \mathbf{G} \cdot \mathbf{u}+\mathbf{n} .
$$

By using eigenvalue decomposition, $\gamma_{m m s e}$ can be then expressed as [9]:

$$
\gamma_{m m s e}=E s \cdot \operatorname{tr}\left[\frac{\Lambda}{(\Lambda+\alpha I)^{2}}\right]=E s \cdot \sum_{k=1}^{N_{r}} \frac{\lambda_{l}}{\left(\lambda_{l}+\alpha\right)^{2}},
$$

with $\lambda_{l}$ being the $l^{\text {th }}$ eignenvalue of $\mathbf{H} \mathbf{H}^{H}$ and $\mathbf{I}$ being the identity matrix. Without the the normalization factor $\alpha$, small eigenvalues would lead to extremely high $\gamma_{m m s e}$ values and, consequently, to extremely reduced signal power at the user equipment (UE) side. While larger $\alpha$ values are more efficient in negating the effect of small eigenvalues, they induce larger multi-user interference. Selecting $\alpha=N_{r} \cdot \sigma^{2}$ provides the optimal trade-off, and maximizes the resulting SINR [9].

Vector Perturbation: To maximize throughput, VP minimizes $\gamma_{v p}$ (and therefore maximizes the received SNR) by perturbing the information vector $\mathbf{u}$ with the perturbation vector $\mathbf{l}$, and then by multiplying it with the pseudo inverse of the channel matrix $\mathbf{G}_{v p}=\mathbf{H}^{H} \cdot\left(\mathbf{H} \cdot \mathbf{H}^{H}\right)^{-1}$. Specifically, all elements of $\mathbf{l}$ are restricted to the form $a+i \cdot b$, with $a$ and $b$ being integers [9]. The set of all eligible perturbation vectors is denoted as $\mathbb{L}$. The precoding vector $\mathbf{s}$ (before power normalization) is given by $\mathbf{s}=\mathbf{G} \cdot(\mathbf{u}-\tau \cdot \mathbf{l})$, with $\tau$ being a positive constant ${ }^{1}$. Then, the vector that maximizes the received SNR can be found by solving the $N_{r}$ dimensional least squares problem [11]:

$$
\mathbf{l}_{\min }=\arg \min _{\tilde{\mathbf{l}} \in \mathbb{L}}\left(\|\mathbf{G} \cdot(\mathbf{u}-\tau \cdot \tilde{\mathbf{l}})\|^{2}\right) .
$$

To fulfil the transmitter's power constraint, the vector $\mathbf{s}$ has to be normalized by $\sqrt{\frac{P_{t}}{\gamma_{v p}}}$, with

$$
\gamma_{v p}=E\left\{\|\mathbf{G} \cdot(\mathbf{u}-\tau \cdot \mathbf{l})\|^{2}\right\} .
$$

In contrast to $\gamma_{m m s e}$, calculating $\gamma_{v p}$ does not have a closed form expression. However, a lower bound of $\gamma_{v p}$, has been provided in [17]

$$
\gamma_{v p} \approx \frac{N_{r} \Gamma\left(N_{r}+1\right)^{1 / N_{r}}}{\left(N_{r}+1\right) \cdot \pi} \cdot \operatorname{det}\left(\mathbf{H} \cdot \mathbf{H}^{H}\right)^{1 / N_{r}},
$$

${ }^{1}$ A detailed discussion on the impact the parameter of the parameter $\tau$ can be found in [9], [21]. 
where $\Gamma()$ denotes the gamma function and $\operatorname{det}(\mathbf{A})$ the determinant of matrix A. In Section V, we validate for the first time in the open literature, that (7) is accurate enough to perform practical rate adaptation. The received vector, for a VP MUMIMO systems is

$$
\begin{aligned}
\mathbf{y}_{v p} & =\mathbf{H} \cdot \sqrt{\frac{P_{t}}{\gamma_{v p}}} \cdot \mathbf{G} \cdot(\mathbf{u}-\tau \cdot \mathbf{l})-\mathbf{n} \\
& =\sqrt{\frac{P_{t}}{\gamma_{v p}}} \cdot(\mathbf{u}-\tau \cdot \mathbf{l})+\mathbf{n} .
\end{aligned}
$$

To recover $\mathbf{u}$, since $\mathbf{l}$ is a complex integer vector, the receiver can utilize the following modulo function with respect to $\tau$

$$
\tilde{\mathbf{y}}_{v p}=\bmod \left[\sqrt{\frac{\gamma_{v p}}{P_{t}}} \cdot \mathbf{y}_{v p}\right]_{\tau}
$$

with $\bmod [a]_{b}$ being the modulo $b$ with respect to $a$. For the remainder of this work $\tau$ is set to

$$
\tau=2 \cdot\left(\left|c_{\max }\right|+\frac{\Delta}{2}\right)
$$

with $\left|c_{\max }\right|$ being the maximum real part that a QAM symbol may have in the selected QAM constellation, and $\Delta$ being the minimum distance between any two constellation points.

Sphere encoders solve (5), by translating the minimization problem into a tree search problem. Specifically, by QRdecomposing the precoding matrix, $\mathbf{G}$ can be expressed as $\mathbf{G}=\mathbf{Q R}$, where $\mathbf{Q}$ is an orthonormal and $\mathbf{R}$ an upper triangular matrix), hence (5) becomes

$$
: \mathbf{l}_{\min }=\arg \min _{\tilde{\mathbf{l}}}\left(\|\mathbf{R} \cdot(\mathbf{u}-\tau \cdot \tilde{\mathbf{l}})\|^{2}\right) .
$$

In order to build a finite search tree, as described in [10], the absolute values of the real and the imaginary parts of any component of the perturbation vector $\mathbf{I}$ have to be limited to integer values smaller or equal to $\mathcal{B}$, with $\mathcal{B}$ being a positive integer. Then, the brunching factor of the SE tree is $|B|=(2 \cdot \mathcal{B}+1)^{2}$ and its height is $N_{r}$. The $k^{t h}$ level of the tree is associated with the $k^{t h}$ element of the the perturbation vector. Each node at level $k$ is associated with a partial perturbation vector $\mathbf{l}_{k}=\left[l\left(N_{r}-k\right), \ldots, l\left(N_{r}\right)\right]$ containing the possible perturbation symbols from the root down to level $k$. Each node is, then, characterized by its partial Euclidean distance $d\left(\mathbf{l}_{k}\right)$ :

$$
d\left(\mathbf{l}_{k}\right)=\left[\hat{s}(k)-\sum_{p=k+1}^{N_{r}} R(k, p) \cdot \tau \cdot l_{k}(p)\right]^{2}+d\left(\mathbf{l}_{k+1}\right),
$$

with $R(k, p)$ being the element of $\mathbf{R}$ at the $k^{t h}$ column and $p^{\text {th }}$ row, $\hat{\mathbf{s}}$ being equal to $\mathbf{R} \cdot \mathbf{u}$ and $d\left(\mathbf{l}_{N_{r}+1}\right)=0$. Then, the VP identification problem translates into finding the leaf node with the smallest $d\left(\mathbf{l}_{1}\right)$.

\section{ViPER SYSTEM DESIGN}

The ViPer MIMO system design consists of (i) The ViPerPhysical Layer (PHY) Processing unit that enables flexible, massively parallel and low-latency VP vector identification. It is an extension of the work in [18], [19] and consists of a new heuristic "metric of promise" that is tailored to the downlink and of a novel "sorted" QR decomposition. (ii) ViPer's Rate Adaptation unit, that is based on the work of [17], enables practical adaptive modulation and coding.

ViPer PHY Processing: Similarly to [18], [19], ViPer has the ability to a priori identify the sphere encoding tree paths that are "most promising" to include the optimal perturbation vector, based on metric which is specifically tailored to the downlink. Similarly to [19], ViPer then processes only these paths. As discussed, the original "metric of promise" (MoP) in [19] is not applicable to the downlink. Therefore, ViPer utilizes a new, heuristic MoP where high values indicate that it is unlikely for the corresponding sphere encoding tree path to be associated with the optimal perturbation vector. To uniquely describe a particular tree path we adopt the concept of position vectors of ([18], [19]). A position vector $\mathbf{p}$ is an $N_{r} \times 1$ dimensional vector, with each of its elements being integer in $[1,|B|]$. The $k^{t h}$ element of $\mathbf{p}$, here denoted as $p(k)$, describes the index of the corresponding node in the $k^{\text {th }}$ level of the sphere encoder tree, when the nodes are sorted in ascending order of their associated partial Euclidean distances.

ViPer's metric of promise: In contrast to the uplink [19] it is extremely challenging to a priori (e.g. at the preprocessing stage) quantify each position vector's likelihood to correspond to the perturbation vector $\mathbf{l}_{\min }$. Instead, for each $\mathbf{p}$ we introduce a lower bound of its corresponding Euclidean distance $d\left(\mathbf{l}_{1}\right)$ across all possible information vectors $\mathbf{u}$. Subsequently, the position vectors with the smallest Euclidean distance bound are considered to be the "most promising" to be associated with the optimal perturbation vector. In other words, ViPer focuses it's processing power on position vectors that can potentially result in very small $d\left(\mathbf{l}_{1}\right)$ values.

Any position vector $\mathbf{p}$ is associated with one particular SE tree path that is comprised of $N_{r}$ nodes (one node for each tree level). All those nodes are associated with one particular perturbation symbol and contribute to the $d\left(\mathbf{l}_{1}\right)$ value for this p. We denote the contribution of the node of the $e^{t h}$ tree level as $\delta_{\mathbf{p}}(e)$. Thus,

$$
d\left(\mathbf{l}_{1}\right)=\sum_{e=1}^{N_{r}} \delta_{\mathbf{p}}(e)
$$

To find a lower bound for $\delta_{\mathbf{p}}(e)$, we first derive an individual lower bound for each $\delta_{\mathbf{p}}(e)$. Using (14), $\delta_{\mathbf{p}}(e)$ becomes:

$$
\delta_{\mathbf{p}}(e)=[R(e, e)]^{2} \cdot \tau^{2} \cdot\left\|\tilde{s}(e)-l_{e}(e)\right\|^{2}
$$

with

$$
\tilde{s}(e)=\left[\frac{\hat{s}(e)-\sum_{k=e+1}^{N_{r}} R(e, k) \cdot \tau \cdot l_{e}(k)}{R(e, e) \cdot \tau}\right]
$$

where $l_{e}(e)$ is the perturbation symbol that is associated with the tree node at level $e$. Theoretically, $l_{e}(e)$ could be identical 
to $\tilde{s}(e)$ (see (17)), which would result in $\delta_{\mathbf{p}}(e)=0$. Then $l_{e}(e)$ will be the closest perturbation symbol to $\tilde{s}(e)$. Otherwise, if $l_{e}(e)$ is not the closest perturbation symbol to $\tilde{s}(e)$, it holds that $\delta_{\mathbf{p}}(e)>0$. Let $l_{1^{s t}}$ denote the perturbation symbol closest to $\tilde{s}(e)$. Then,

$$
\delta_{\mathbf{p}}(e) \geq[R(e, e)]^{2} \cdot \tau^{2} \cdot \frac{\left\|l_{1^{s t}}-l_{e}(e)\right\|^{2}}{2} .
$$

In [19] it has been shown that for integer lattices the distance between a lattice point and its $n^{\text {th }}$ closet neighbour approximately scales with $\sqrt{n}$. By applying the same observation here, we have that

$$
\begin{aligned}
\delta_{\mathbf{p}}(e) & \geq[R(e, e)]^{2} \cdot \tau^{2} \cdot \frac{\left\|\ell-l_{e}(e)\right\|^{2}}{2} \\
& \approx[R(e, e)]^{2} \cdot \tau^{2} \cdot \frac{(\sqrt{n-1})^{2}}{2} .
\end{aligned}
$$

From the definition of position vectors it follows that if $l_{e}(e)$ is the $n^{\text {th }}$ closest perturbation symbol to $\tilde{s}(e)$ then $p(e)=n$. Therefore, by using (19) and (15), a lower bound of $d\left(\mathbf{l}_{1}\right)$ can be calculated as:

$$
=\sum_{e=1}^{N_{r}} \delta_{\mathbf{p}}(e) \geq \sum_{e=1}^{N_{r}}[R(e, e)]^{2} \cdot \tau^{2} \cdot \frac{\sqrt{p(e)-1}^{2}}{2} .
$$

Considering that $\tau^{2} / 2$ is a constant factor we omit it form the final definition of the MoP and therefore

$$
\mathcal{M o P}(\mathbf{p})=\sum_{e=1}^{N_{r}}\left\{[R(e, e)]^{2} \cdot(p(e)-1)\right\} .
$$

To find the position vectors with the lowest MoPs a K-best tree search can be used as in [18] or alternatively the tree structure and search of [19] can be utilized.

After finding the position vectors with the lowest MoPs, the positions vectors are processed in parallel as in [19]. Finally, the perturbation vector associated with the position vector that has the smallest $d\left(\mathbf{l}_{1}\right)$ metric is chosen.

ViPer's sorted $R Q$ decomposition: In the MIMO uplink, it is well known that changing (permuting) the detection order of the users by means of sorted $Q R$ decomposition (SQRD) can maximize the diagonal elements of the $\mathbf{R}$ matrix (when $\mathbf{H}=\mathbf{Q R}$ ) resulting in improved detection efficiency [20], [19]. To the best of our knowledge no VP-based precoder has leveraged a similarly improved processing in order to increase VP's vector identification efficiency.

Normally, in order to achieve such a sorted order, a precoder should first calculate the pseudo inverse of the channel matrix to get $\mathbf{G}_{v p}$ and then execute SQRD for the matrix $\mathbf{G}_{v p}$. Both operations (i.e. matrix inversion and $\mathrm{SQRD}$ ) are of complexity of $\mathcal{O}\left(N_{r}^{2} \cdot N_{t}\right)$. Therefore, their sequential execution would significantly affect the already large pre-processing latency of VP. Instead, ViPer performs RQ decomposition directly on the channel matrix $\mathbf{H}$ and applies a modified version of [22]. In contrast to the traditional SQRD, the proposed method permutes the rows (and not the columns) of $\mathbf{H}$. The pseudo code for this proposed sorted RQ-decomposition is presented in algorithm 1. The RQ-decomposition results in an $N_{r} \times N_{r}$ upper triangular matrix $\mathbf{R}_{H}$ and an $N_{r} \times N_{t}$ matrix $\mathbf{Q}_{H}$, with

$$
\mathbf{H}=\mathbf{R}_{H} \cdot \mathbf{Q}_{H}
$$

We note that the matrix $\mathbf{R}_{H}$ is related to the channel matrix $\mathbf{H}$ and not to the precoding matrix $\mathbf{G}_{v p}$ as would be required to perform SE. Using (22),

$$
\mathbf{G}_{v p}=\mathbf{H}^{H}\left(\mathbf{H} \mathbf{H}^{H}\right)=\mathbf{Q}_{H}^{H} \cdot \mathbf{R}_{H}^{H} \cdot\left(\mathbf{R}_{H} \mathbf{Q}_{H} \mathbf{Q}_{H}^{H} \mathbf{R}_{H}^{H}\right)^{-1}
$$

By design, matrix $\mathbf{Q}_{H}$ fulfills the property $\mathbf{Q} \mathbf{Q}^{H}=\mathbf{I}$, which simplifies (23) to

$$
\mathbf{G}_{v p}=\mathbf{Q}_{H} \cdot \mathbf{R}_{H} \cdot\left(\mathbf{R}_{H} \mathbf{R}_{H}^{H}\right)^{-1}=\mathbf{Q}_{H}^{H} \cdot\left(\mathbf{R}_{H}\right)^{-1}
$$

The precoding matrix can be then expressed as $\mathbf{G}_{v p}=$ $\mathbf{Q}_{V i P} \mathbf{R}_{V i P}$, with $\mathbf{Q}_{V i P}=\mathbf{Q}_{H}^{H}$ and $\mathbf{R}_{V i P}=\left(\mathbf{R}_{H}\right)^{-1}$, which is an appropriate structure in order to preform VP. We note that inverting the upper triangular matrix $\mathbf{R}_{H}$ is significantly less complex than inverting the channel matrix $\mathbf{H}$.

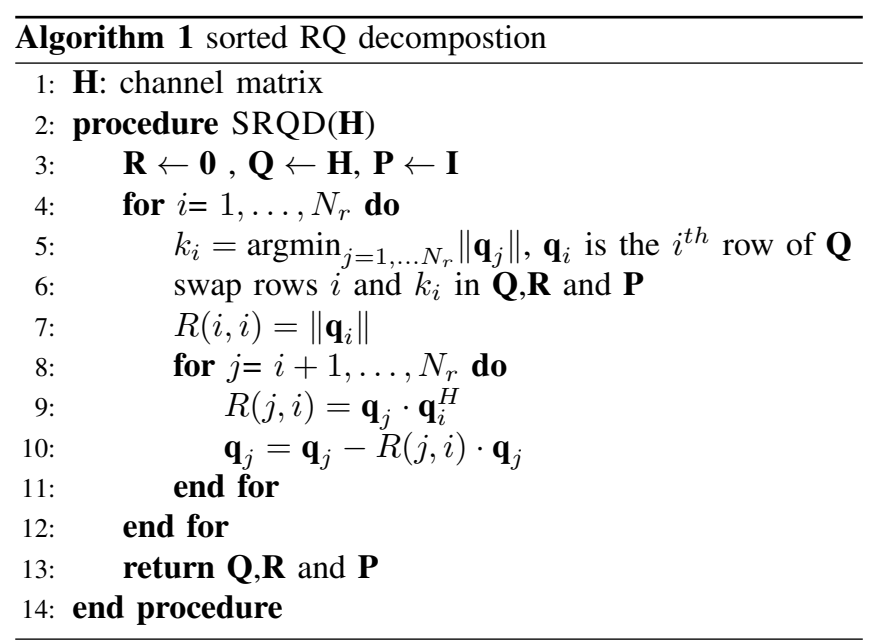

ViPer Rate Adaptation: To translate the capacity gains of ViPer into actual throughput gains, a rate adaption technique is required. ViPer's rate adaptation leverages the lower bound on $\gamma_{v p}$ (7) to estimate the SNR of the user equipment (UE) at the AP when transmitting VP precoded information. By repurposing, the $\mathrm{QR}$ decomposition of the precoding matrix $\mathbf{G}_{v p}=\mathbf{Q}_{V i P} \mathbf{R}_{V i P}$, we simplify (7) as

$$
\tilde{\gamma}_{v p} \approx \frac{N_{r} \Gamma\left(N_{r}+1\right)^{1 / N_{r}}}{\left(N_{r}+1\right) \cdot \pi} \cdot\left(\prod_{j=1}^{N_{r}}\left[R_{V i P}(j, j)\right]^{2}\right)^{1 / N_{r}}
$$

Since the first factor in (25) depends only on the number of concurrent information streams, it can be precalculated and saved in a look-up table.

Equation (25) has been derived for theoretical signals, with their real and imagery parts being equally distributed in [-0.5, 0.5]. In order for (25) to be applicable to standard QAMmodulation, the expression has to be normalized as

$$
\gamma_{v p}=\tau^{2} \cdot \tilde{\gamma}_{v p}
$$




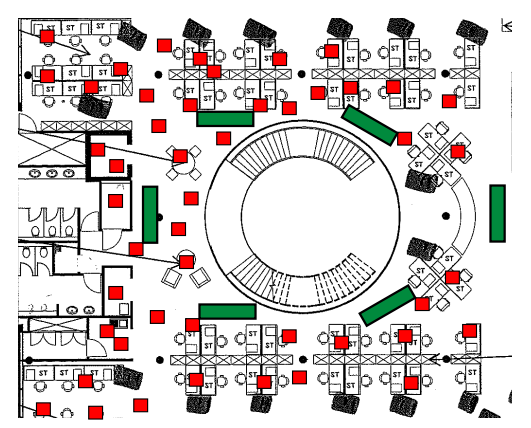

Fig. 1. Testbed floorplan: 8-antenna APs (rectangles), single-antenna UEs (squares) .

and the UEs' SNR to be approximated as:

$$
S N R \approx \frac{E s}{\sigma^{2} \cdot \tilde{\gamma}_{v p}}=\frac{E s}{\sigma^{2} \cdot \tau^{2} \cdot \tilde{\gamma}_{v p}} .
$$

Since $\tau$ depends on the selected modulation (see (12)), the SNR expression (27) can not directly be used to determine the optimal transmission rate. Instead we utilize $\tilde{\gamma}_{v p}$ and a slightly modified SNR mapping. Let $b(n)$ be the SNR for which the the $n^{\text {th }}$ transmission rate reaches a reasonably small block error probability (e.g., 10\%). Then, the ViPer's rate adaption unit selects the $n^{\text {th }}$ transmission rate if and only if

$$
b(n) \cdot \tau_{n}^{2} \leq \frac{E s}{\sigma^{2} \cdot \tilde{\gamma}_{v p}} \leq b(n+1) \cdot \tau_{n+1}^{2},
$$

where $\tau_{n}$ denotes the value of the parameter $\tau$ that corresponds to the QAM-constellation utilized in the $n^{\text {th }}$ transmission rate (see (12)). Similar to traditional SNR mapping, the term $b(n)$. $\tau_{n}^{2}$ can be precalculated for all supported transmission rates and stored in a look-up table.

\section{Evaluation}

In order to evaluate ViPer's throughput performance we employ the BEEcube MegaBEE MIMO prototyping hardware and MATLAB 2017b. Our OFDM system operates at a frequency of $2.6 \mathrm{GHz}$ with $20 \mathrm{MHz}$ bandwidth and a subcarrier spacing of $15 \mathrm{KHz}$. The AP antenna is a linear array of 8 patch antennas with half wavelength antenna spacing. Fig. 1 depicts the positions of the AP and the UEs during the experimental evaluation. Both, the employed LDPC codes and the rate matching algorithm follow the latest 3GPP standard [23] and are implemented using MATLAB's 5G toolbox. Our system supports 9 transmission modes with rates between 0.5 bits/symbol and 5 bits/symbol. All implemented VP-based precoding methods choose the perturbation vector from an integer lattice with 9 points $^{2}$.

Experimental Evaluation: In over-the-air experiments we investigate the throughput performance of ViPer, FCSD, SE and MMSE precoding. To emulate a simple user selection approach we assume that all users belong to the same SNR regime. Here we examine two SNR regimes. An intermediate

\footnotetext{
${ }^{2}$ Both the real and imaginary parts of the perturbation symbols take one of the three integer values $\{-1,0,1\}$.
}

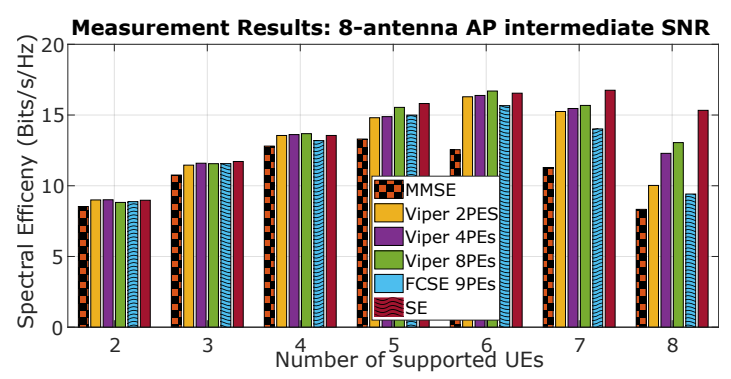

Fig. 2. Achievable throughput of ViPer, FCSE, SE and MMSE as a function of the number of served users in the intermediate SNR regime.

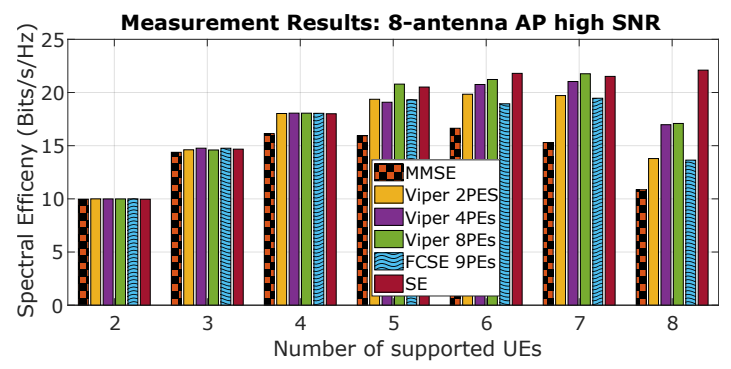

Fig. 3. Achievable throughput of ViPer, FCSE, SE and MMSE as a function of the number of served users in the high SNR regime.

SNR regime $(12.5 \mathrm{~dB}$ to $17.5 \mathrm{~dB})$ and a high SNRs regime (17.5 dB to $22.5 \mathrm{~dB})$.

Fig. 2 shows the achievable net-throughput for all implemented MIMO precoders as a function of the number of concurrently served UEs, at the intermediate SNR regime. As also shown in [7], MMSE precoding is efficient only when the number of supported UEs is significantly smaller than the number of AP antennas. If five or more users are supported, MMSE pre-coding results in a noticeable throughput loss compared to the VP-based methods. When employing 9 PEs (FCSE's minimum requirement) and by using each PE to examine a single $\mathrm{SE}$ tree path (to minimize processing latency) FCSE's peak throughput is $15 \%$ higher than that of MMSE. Still, its throughput is lower than ViPer's, and FCSE requires $4 \times$ the amount of ViPer's PEs. We note that for up to 6 concurrently supported UEs, ViPer nearly achieves the throughput of optimal sphere encoding, by employing only two PEs. Below we will show that ViPer can approach the throughput of SE for any number of supported UEs by increasing the number of employed PEs. Nevertheless, the peak performance of ViPer using only two PEs is $25 \%$ higher than that of MMSE and only 3\% smaller than that of "optimal" SE.

Fig. 3 depicts the throughput results in the high SNR regime. In general, the same trends with the intermediate SNR hold. At high SNRs and with 8 available PEs, ViPer matches the performance of optimal SE for up to 7 concurrently supported UEs. In this configuration the peak performance of of ViPer is $31 \%$ larger than that of MMSE and only $1.5 \%$ smaller than that of SE.

Simulation Evaluation: To illustrate the impact of the 


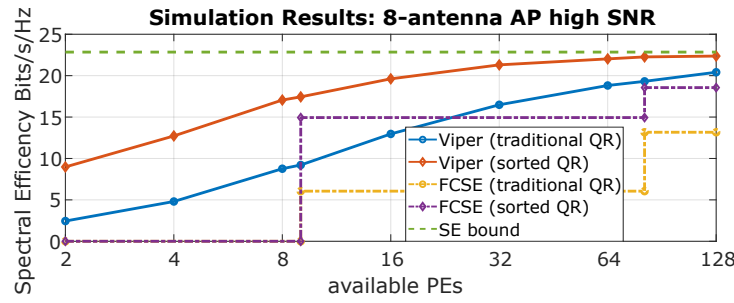

Fig. 4. Achievable throughput of ViPer and FCSE with 8 concurrently served users as function of the available PEs in the high SNR regime.

proposed RQ decomposition as well as the number of utilized PEs on the throughput performance of ViPer and FCSE, we additionally perform trace-driven-simulations using the previously measured channels. Fig. 4 shows the achievable throughput as a function of the available PEs in the high SNR regime $^{3}$. It is evident that ViPer, in contrast to FSCE, is able to consistently increase network throughput by increasing the number of PEs and eventually converges to the performance of "optimal" SE regardless of the employed QR decomposition. In contrast, traditional FCSE without the proposed sorted RQ and 81 PEs (for FSCE the number of PEs should be an integer power of the size of the perturbation constellation) reaches only a throughput of $13.17 \mathrm{bits} / \mathrm{sec} / \mathrm{Hz}$, which is only $57 \%$ of the throughput of the optimal SE. Furthermore, ViPer with 6 PEs outperforms FSCE by only utilizing one tenth of the PEs.

The impact of ViPer's RQ decomposition is particular large for small numbers of PEs. For example, if 9 PEs are employed, both ViPer's and FCSE's achievable throughput is increased by $244 \%$ and $189 \%$, respectively. Finally, the processing complexity of the optimal SE (implemented by means of Geosphere [10]) is reduced from 1983 visited nodes to 417 when replacing traditional QR decomposition with the ViPer's RQ-decomposition.

\section{CONCLUSION}

In this paper we presented ViPer, the first practical MIMO downlink system design, that can efficiently be realized in a flexible and massively parallel manner. In our over-the-airexperiments we show that ViPer can exceed the throughput of linear methods by more than $30 \%$, and exhibit similar latency. Furthermore, ViPer can match the throughput of state-of-theart parallel MIMO precoders, with an order of magnitude fewer processing elements.

\section{ACKNOWLEDGMENT}

This work has been supported by the UK's Engineering and Physical Sciences Research Council (EPSRC Ref. EP/M029441/1), and the DCMS Initial 5G Testbeds and Trials project "AutoAir". The Authors would also like to thank the members of University of Surrey 5GIC (http://www.surrey.ac.uk/5GIC) for their support.

\footnotetext{
${ }^{3}$ Since in trace-driven simulations we assume no RF imperfections, the high SNR regime here refers to SNR values between $16.5 \mathrm{~dB}$ and $21.5 \mathrm{~dB}$ in order to show comparable throughput results to the over-the-air experiments.
}

\section{REFERENCES}

[1] J. G. Andrews and et al., "What will 5G be?" IEEE J. Sel. Areas Commun., vol. 32, no. 6, pp. 1065-1082, 2014.

[2] A. Ghosh and et al., "Millimeter-wave enhanced local area systems: A high-data-rate approach for future wireless networks," IEEE J. Sel. Areas Commun., vol. 32, no. 6, pp. 1152-1163, 2014.

[3] 3GPP, "Study on New Radio (NR) Acceess Technology; Physical Layer Aspects," 3rd Generation Partnership Project (3GPP), Technical Report (TR) 38.801, 03 2017, version 2.0.0.

[4] "Introduction to 802.11ax High-Efficiency Wireless," National Instruments, Tech. Rep., 062017.

[5] I. F. Akyildiz, S. Nie, S.-C. Lin, and M. Chandrasekaran, "5G roadmap: 10 key enabling technologies," Comput. Networks, vol. 106, pp. 17-48, 2016.

[6] S. Malkowsky and et al., "The worlds first real-time testbed for massive MIMO: Design, implementation, and validation," IEEE Access, vol. 5, pp. 9073-9088, 2017.

[7] Q. Yang and et al., "Bigstation: enabling scalable real-time signal processingin large mu-mimo systems," in Proc. ACM SIGCOMM, vol. 43, no. 4. ACM, 2013, pp. 399-410.

[8] T. Yoo and A. Goldsmith, "On the optimality of multiantenna broadcast scheduling using zero-forcing beamforming," IEEE J. Sel. Areas Commun., vol. 24, no. 3, pp. 528-541, 2006.

[9] C. B. Peel, B. M. Hochwald, and A. L. Swindlehurst, "A vector-perturbation technique for near-capacity multiantenna multiuser communication-part i: channel inversion and regularization," IEEE Trans. Commun., vol. 53, no. 1, pp. 195-202, 2005.

[10] K. Nikitopoulos, J. Zhou, B. Congdon, and K. Jamieson, "Geosphere: Consistently turning MIMO capacity into throughput," in Proc. ACM SIGCOMM, vol. 44, no. 4, 2014, pp. 631-642.

[11] B. M. Hochwald, C. B. Peel, and A. L. Swindlehurst, "A vector-perturbation technique for near-capacity multiantenna multiuser communication-part ii: Perturbation," IEEE Trans. Commun., vol. 53, no. 3, pp. 537-544, 2005.

[12] M. Costa, "Writing on dirty paper," IEEE Trans. Commun., vol. 29, no. 3, pp. 439-441, 1983.

[13] H. Harashima and H. Miyakawa, "Matched-transmission technique for channels with intersymbol interference," IEEE Trans. Commun., vol. 20, no. 4, pp. 774-780, 1972.

[14] G. Georgis, K. Nikitopoulos, and K. Jamieson, "Geosphere: An exact depth-first sphere decoder architecture scalable to very dense constellations," IEEE Access, vol. 5, pp. 4233-4249, 2017.

[15] R. Courtland, "Transistors could stop shrinking in 2021," IEEE Spectrum, vol. 53, no. 9, pp. 9-11, 2016.

[16] M. Mohaisen and K. Chang, "Fixed-complexity sphere encoder for multi-user MIMO systems," J. of Commun. and Networks, vol. 13, no. 1, pp. 63-69, 2011.

[17] D. J. Ryan, I. B. Collings, I. V. L. Clarkson, and R. W. Heath, "Performance of vector perturbation multiuser MIMO systems with limited feedback," IEEE Trans. Commun., vol. 57, no. 9, 2009.

[18] K. Nikitopoulos, D. Chatzipanagiotis, C. Jayawardena, and R. Tafazolli, "Multisphere: Massively parallel tree search for large sphere decoders," in Proc. Global Commun. Conf. (GLOBECOM). IEEE, 2016, pp. 1-6.

[19] C. C. M. Husmann, G. Georgis, K. Nikitopoulos, and K. Jamieson, "Flexcore: Massively parallel and flexible processing for large mimo access points," in Proc. of the 14th USENIX Symp. on Networked Syst. Design and Implementation (NSDI), 2017, pp. 197-211.

[20] L. G. Barbero and J. S. Thompson, "A fixed-complexity MIMO detector based on the complex sphere decoder," in Proc. Signal Processing Advances in Wireless Communications, 2006. SPAWC'06. IEEE 7th Workshop on. IEEE, 2006, pp. 1-5.

[21] C. NYuen and B. Hochwald, "How to gain $1.5 \mathrm{db}$ in vector precoding." in Proc. Global Commun. Conf. (GLOBECOM). IEEE, 2006, pp. 1-6.

[22] D. Wubben, R. Bohnke, J. Rinas, V. Kuhn, and K.-D. Kammeyer, "Efficient algorithm for decoding layered space-time codes," Electron. lett., vol. 37, no. 22, pp. 1348-1350, 2001

[23] 3GPP, "Multiplexing and channel coding," 3rd Generation Partnership Project (3GPP), Technical Report (TR) 38.221, 08 2017, version 0.2.1. 